\title{
Poloxamer 407/TPGS mixed micelles for delivery of gambogic acid to breast and multidrug-resistant cancer
}

\section{Vipin Saxena}

M Delwar Hussain

Department of Pharmaceutical Sciences, Irma Lerma Rangel College of Pharmacy, Texas A\&M Health Science Center, Kingsville, TX, USA
Correspondence: M Delwar Hussain I0I0 West Avenue B, Kingsville, Texas 78363, USA

Tel + I 36I 5934310

Fax + I 36I 5934303

Email hussain@pharmacy.tamhsc.edu
This article was published in the following Dove Press journal:

International Journal of Nanomedicine

13 February 2012

Number of times this article has been viewed

Background: Delivery of a high concentration of anticancer drugs specifically to cancer cells remains the biggest challenge for the treatment of multidrug-resistant cancer. Poloxamers and D- $\alpha$-Tocopheryl polyethylene glycol 1000 succinate (TPGS) are known inhibitors of P-glycoprotein (P-gp). Mixed micelles prepared from Poloxamer 407 and TPGS may increase the therapeutic efficacy of the drug by delivering high concentrations inside the cells and inhibiting P-gp. Gambogic acid (GA) is a naturally derived novel anticancer agent, but poor solubility and toxic side effects limit its use. In this study, we have developed Poloxamer 407 and TPGS mixed micelle-encapsulating GA for the treatment of breast and multidrug-resistant cancer.

Methods: GA-loaded Poloxamer 407/TPGS mixed micelles were prepared using a thin film hydration method, and their physicochemical properties were characterized. Cellular accumulation and cytotoxicity of the GA-loaded Poloxamer 407/TPGS mixed micelles were studied in breast cancer cells, MCF-7 cells, and multidrug-resistant NCI/ADR-RES cells.

Results: The diameter of GA-loaded Poloxamer 407/TPGS mixed micelles was about $17.4 \pm 0.5 \mathrm{~nm}$ and the zeta potential $-13.57 \mathrm{mV}$. The entrapment efficiency of GA was $93.1 \% \pm 0.5 \%$ and drug loading was about $9.38 \% \pm 0.29 \%$. Differential scanning calorimetry and X-ray powder diffraction studies confirmed that GA is encapsulated by the polymers. The in vitro release studies showed that mixed micelles sustained the release of GA for more than 4 days. Results from cellular uptake studies indicated that GA-loaded Poloxamer 407/TPGS mixed micelles had increased cellular uptake of GA in NCI/ADR-RES cells. Cytotoxicity of GA-loaded Poloxamer 407/TPGS mixed micelles was found to be 2.9 times higher in multidrug-resistant NCI/ADR-RES cells, and 1.6 times higher in MCF-7 cells, as compared with unencapsulated GA.

Conclusion: This study suggests that Poloxamer 407/TPGS mixed micelles can be used as a delivery system for GA to treat breast and multidrug-resistant cancer.

Keywords: gambogic acid, Poloxamer 407, TPGS, P-glycoprotein, multidrug resistance, breast cancer

\section{Introduction}

Multidrug resistance (MDR) is the phenomenon of simultaneous resistance to many structurally unrelated cytotoxic agents, and is one of the most formidable challenges in the field of cancer therapy. A common mechanism of MDR is the active export of drugs from cancer cells by the overexpression of P-glycoprotein (P-gp) and other adenosine triphosphate (ATP)-binding cassette transporters. ${ }^{1,2}$ The use of Poloxamer block copolymers as a drug delivery vehicle to treat MDR cancers is a rapidly developing area for cancer chemotherapy. Poloxamers have been shown to reverse MDR by inhibiting the P-gp pump. ${ }^{3,4}$ 
Gambogic acid (GA) is the principal active ingredient of gamboge, the resin found in trees of the genus Garcinia. GA possesses diverse biological properties such as anti-inflammatory, antioxidant, antiviral, anti-infectious, and neuroprotective effects. ${ }^{5,6}$ Additionally, GA inhibits the growth of different types of cancer including gastric cancer, lung carcinoma, prostate cancer, melanoma, hepatocarcinoma, and breast cancer. ${ }^{7-11}$

Several possible mechanisms are proposed for the anticancer effect of GA, such as induction of apoptosis, microtubule depolymerization, and downregulation of telomerase activity. ${ }^{7,12,13} \mathrm{Gu}$ et $\mathrm{al}^{7}$ showed that GA can induce apoptosis in MCF-7 cancer cells through upregulation of p53 and downregulation of Bcl-2 proteins. GA also depolymerized microtubules and elevated the phosphorylation levels of JNK1 and p38, which caused G2/M cell cycle arrest and apoptosis in MCF-7 cells. ${ }^{12}$ GA significantly inhibited the adhesion, migration, and invasion of the cancer cells in vitro. ${ }^{12,14}$ Wang et a $1^{15}$ describe the potential role of GA to reverse docetaxel resistance though downregulation of survivin in gastric cancer cells. Survivin might play a key role in MDR in the presence of P-gp, and this might represent a novel strategy for modulating MDR in cancer cells. ${ }^{2}$ Recently, a selective apoptotic induction of GA on cancer cells compared with normal cells suggests GA might be an effective anticancer drug candidate with low toxicity to normal cells. ${ }^{16}$

The poor water solubility of GA has limited its use. Several approaches have been used to improve the solubility of the GA, such as conjugation with L-arginine or use of polyoxylated castor oil (Cremophor EL ${ }^{\circledR}$; BASF SE, Ludwigshafen, Germany),${ }^{17}$ but lethal allergic reaction and ulceration was observed with L-arginine containing formulations. The Cremophor EL-containing formulation had several side effects such as cardiotoxicity, nephrotoxicity, neurotoxicity, and hypersensitivity reactions. ${ }^{18}$

Polymeric micelle systems were recognized as one of the most promising strategies to deliver poorly soluble anticancer drugs. ${ }^{19-22}$ Polymeric micelles made of amphiphilic copolymers consist of a core shell structure formed by self-assembly of a hydrophobic block as an internal core and a hydrophilic block as a surrounding shell. Poorly soluble drugs can be made soluble within the hydrophobic inner core of a micelle and hydrophilic shell interface the biological media. ${ }^{23}$ As a result, micelles can substantially improve solubility and bioavailability of various hydrophobic drugs. The nanometer size $(<100 \mathrm{~nm})$ of micelles allows higher accumulation and delivery of encapsulated anticancer drugs, via the enhanced permeability and retention effect. ${ }^{24}$

Among a number of natural and synthetic polymers used to form polymeric micelles, Poloxamer 407, a US Food and Drug Administration-approved polymer, is the most attractive due to its biocompatibility, biodegradability, and low toxicity. Poloxamer block copolymer consists of ethylene oxide (EO) and propylene oxide (PO) blocks that are arranged in a basic $\mathrm{EO}_{\mathrm{x}}-\mathrm{PO}_{\mathrm{y}}-\mathrm{EO}_{\mathrm{x}}$ structure. The $\mathrm{PO}$ core can serve as a "pool" and the hydrophobic drug can be incorporated into the hydrophobic PO core, while the hydrophilic corona maintains the dispersion stability of Poloxamer micelles. Incorporation of drugs into micelles leads to increased solubility, metabolic stability, and circulation time of the drug..$^{25,26}$

D- $\alpha$-Tocopheryl polyethylene glycol 1000 succinate (TPGS), a derivative of natural vitamin $\mathrm{E}$ ( $\alpha$-tocopherol) and polyethylene glycol 1000, has been widely applied in the food and drug industry as solubilizer, absorption enhancer, and a vehicle for lipid-based drug delivery formulations. It has also been found that TPGS enhances the solubility, inhibits P-gp-mediated multidrug resistance, and increases the oral bioavailability of anticancer drugs. ${ }^{27}$ It was also reported that succinate esters of vitamin $\mathrm{E}$ behave as potent proapoptotic agents selective for cancer cells. ${ }^{28}$ Mixed micelles manifest synergistic properties, such as increased micelle stability and drug-loading efficiency, superior to those of the individual components. ${ }^{29}$

We hypothesize that GA-loaded mixed micelles made of Poloxamer 407 and TPGS may allow a better nanoformulation, more stability, and higher anticancer efficiency in resistant breast cancer. GA-loaded mixed Poloxamer 407/TPGS micelles were prepared and their physical properties were characterized. The in vitro cellular uptake and cytotoxicity against breast cancer cells, MCF-7, and multidrug resistant NCI/RES-ADR cells were studied.

\section{Methods and materials}

Poloxamer 407 was obtained as a gift from BASF (Tarrytown, NY), TPGS was purchased from Sigma-Aldrich (St Louis, MO). GA was purchased from 2A PharmaChem (Lisle, IL). All other reagents and buffer solutions were of analytical grade. Distilled and deionized (DI) water was used in all experiments.

\section{Preparation of micelles}

GA-loaded Poloxamer 407/TPGS mixed micelles were prepared by thin film hydration method as described earlier in Zhang et al. ${ }^{26}$ Briefly, GA (9 mg), Poloxamer 407 (68 mg), 
and TPGS (23 mg) were dissolved in $3 \mathrm{~mL}$ acetonitrile in a round bottom flask. The solvent was evaporated by rotary evaporation at $40^{\circ} \mathrm{C}$ for 1 hour to obtain a solid GA/copolymer matrix. Residual acetonitrile remaining in the film was removed under vacuum overnight at room temperature. The resultant thin film was hydrated with $4 \mathrm{~mL}$ water at $40^{\circ} \mathrm{C}$ for 30 minutes to obtain a micelle solution, which was then filtrated through $0.2 \mu \mathrm{m}$ filter membrane to remove the unincorporated GA aggregates, followed by lyophilization. Empty Poloxamer 407/TPGS mixed micelles were also prepared as described above, excepting the addition of GA.

\section{Critical micelle concentration (CMC) determination}

To determine the CMC of binary mixture of Poloxamer 407/ TPGS in DI water, an ultraviolet-visible (UV-Vis) spectroscopy method was used, using iodine as a hydrophobic probe. ${ }^{30}$ The KI/ $\mathrm{I}_{2}$ standard solution was prepared by dissolving $0.5 \mathrm{~g}$ of iodine and $1 \mathrm{~g}$ of potassium iodide in $50 \mathrm{~mL}$ DI water. Samples of polymer solution with concentrations ranging from $0.00001 \%$ to $0.1 \%$ were prepared. To each of the Poloxamer 407/TPGS binary mixture (molar ratio 3:7) solutions, $25 \mu \mathrm{L} \mathrm{KI} / \mathrm{I}_{2}$ standard solution was added. The mixtures were incubated for 12 hours in a dark room at room temperature before measurement. The ultraviolet absorbance value of varying polymer concentrations at $366 \mathrm{~nm}$ was measured using a UV-Vis spectrometer (DU 800; Beckman Coulter, Indianapolis, IN). Experiments were performed in triplicate. The absorption intensity was plotted against the logarithm of polymer concentration. The $\mathrm{CMC}$ values correspond to the concentration of the polymer at which the sharp increase in absorbance is observed.

\section{Particle size and zeta potential analysis}

Particle mean size and size distribution were measured by the dynamic light scattering method using a Brookhaven Particle Size/Zeta Potential analyzer (ZetaPALS, Brookhaven Instruments, Holtsville, NY). The analyses were performed with $5 \mathrm{~mW}$ He-Ne laser $(632.8 \mathrm{~nm})$ at a scattering angle of $90^{\circ}$ at $25^{\circ} \mathrm{C}$. Each freeze dried or freshly prepared sample was diluted to the appropriate concentration using DI water. The reported experimental result of each sample was expressed as a mean size \pm standard deviation (SD) for three separate experiments.

\section{Drug loading and encapsulation efficiency}

Drug loading (DL\%) and encapsulation efficiency (EE\%) were determined by UV-Vis-spectrophotometry at maximum absorbance of $361 \mathrm{~nm}$. The micellar solution was suitably diluted with acetonitrile prior to determination. DL\% and $\mathrm{EE} \%$ were calculated using following equations: ${ }^{26}$

$$
\begin{aligned}
\mathrm{DL} \%= & \text { Weight of the drug in micelles } \\
& / \text { Weight of the feeding polymer and drug } \\
& \times 100
\end{aligned}
$$

$\mathrm{EE} \%=$ Weight of the drug in micelles /Weight of the feeding drug $\times 100$

\section{Differential scanning calorimetry (DSC)}

The physical state of GA encapsulated in the mixed micelles and the free GA were examined by DSC using DSC Q200 (TA Instruments, New Castle, DE) under liquid nitrogen at a flow rate of $50 \mathrm{~mL}$ per minute. Then, $5 \mathrm{mg}$ of the sample was heated from $0^{\circ} \mathrm{C}$ to $300^{\circ} \mathrm{C}$ at a temperature-rising speed of $10^{\circ} \mathrm{C}$ per minute.

\section{X-ray powder diffraction analysis (XRPD)} XRPD experiments were performed from $2^{\circ}$ to $40^{\circ}$ ( $2 \theta$ angle $)$ using a D8 X-Ray diffractometer system (Bruker AXS, Madison, WI) with a copper anode source $(\mathrm{K} \alpha, \lambda=1.5406 \AA)$ with a filtered flat lithium fluoride secondary beam monochromator. The divergence, receiver, and detector slit widths were $2 \mathrm{~mm}$; the scatter slit width was $0.6 \mathrm{~mm}$.

\section{In vitro drug release}

To create a pseudo-sink condition, the in vitro release behaviors of GA from mixed micelles were monitored in phosphate-buffered saline (PBS) $(\mathrm{pH} 7.4)$ with $0.5 \%$ Tween $^{\circledR} 80$ (ICI Americas Inc, Wilmington, DE). ${ }^{26}$ Briefly, $10 \mathrm{mg}$ of GA-loaded mixed micelles was introduced into a dialysis membrane bag (MWCO 6000-8000 Da), and the sealed dialysis bag was incubated in $20 \mathrm{~mL}$ of release media into an orbital shaker (VWR International, LLC, Suwanee, GA) at $37^{\circ} \mathrm{C}$ and $100 \mathrm{rpm}$. At predetermined time intervals, a sample was withdrawn and complete release media was replaced with fresh release media. The concentration of GA in a sample was measured by the DU $800 \mathrm{UV}$-Vis spectrophotometer at maximum absorbance of $360 \mathrm{~nm}$ and the cumulative release percentage was calculated. For comparison, the release of GA from dimethyl sulfoxide solution was conducted under the same conditions. ${ }^{31}$

\section{Cell culture}

Breast cancer MCF-7 cells were obtained from the American Type Culture Collection (ATCC; Manassas, VA) and 
multidrug-resistant NCI/ADR-RES cells were obtained from the National Cancer Institute (Bethesda, MD). Cells were cultured in RPMI 1640 media supplemented with $10 \%$ fetal bovine serum and 1\% penicillin/streptomycin (all from Mediatech, Manassas, VA).

\section{Cellular internalization study}

MCF-7 and NCI/ADR-RES cells grown on two-well LabTek chambered glass slides (Nalge Nunc International, Rochester, NY) at a density of $5 \times 10^{4}$ cells/well and incubated for 24 hours. One milliliter of media containing free GA or GA-loaded mixed micelles equivalent to $1 \mu \mathrm{M}$ of GA was added to the cells. After incubation for 4 hours, cells were washed three times with ice cold PBS, fixed with $4 \%$ paraformaldehyde. A cover slip was mounted on the slide and the slide was photographed using a Nikon Eclipse T $i$ (Nikon Instruments Inc, Melvill, NY) confocal laser scanning microscope (CLSM).

\section{In vitro cytotoxicity study}

In vitro cytotoxicity of GA-loaded mixed micelles was determined using 3-(4,5-Dimethylthiazol-2-yl)-2,5diphenyltetrazolium bromide (MTT) assay. Briefly, MCF-7 and NCI/ADR-RES cells were transferred to 96-well tissue culture plates at a density of $2 \times 10^{3}$ cells per well and $5 \times 10^{3}$ cells per well, respectively, 24 hours prior to treatment. The medium was then replaced with fresh medium containing free GA, GA-loaded micelles, and blank micelles at different concentrations. The culture medium without any drug formulation was used as the control. After 72 hours of incubation at $37^{\circ} \mathrm{C}$ and $5 \% \mathrm{CO}_{2}$, media was removed and cells were washed twice with sterile PBS. Then $50 \mu \mathrm{L}$ of MTT solution $(0.5 \mu \mathrm{g}$ per $\mathrm{mL})$ was added to each well and further incubated for 4 hours at $37^{\circ} \mathrm{C}$. Medium was removed and $100 \mu \mathrm{L}$ dimethyl sulfoxide was added to each well to dissolve the purple formazan crystal converted from MTT. Cell viability was assessed by absorbance at $570 \mathrm{~nm}$ measured on a NOVOstar micro plate reader (BMG Labtech Inc, Cary, NC) and the concentration at which $50 \%$ of growth is inhibited $\left(\mathrm{IC}_{50}\right)$ was calculated by GraphPad Prism 5.0 (GraphPad Software, La Jolla, CA).

\section{Statistical analysis}

Data are represented as means \pm SD from at least three separate experiments. Statistical significance was assessed using Student's $t$-test. Differences were considered significant at $P<0.05$.

\section{Results and discussion CMC determination}

The CMC of the micelles influences their in vitro and in vivo stability. Low CMC values of Poloxamer 407/TPGS binary mixture underlie high stability of Poloxamer 407/ TPGS mixed micelles in solutions upon dilution. In this study, the formation of micelles was monitored by using iodine (I) as a hydrophobic probe. Solubilized $\mathrm{I}_{2}$ prefers to participate in the hydrophobic microenvironment of Poloxamer copolymer, causing the conversion of $\mathrm{I}_{3}^{-}$to $\mathrm{I}_{2}$ from the excess potassium iodide (KI) in the solution. The absorbance intensity of $\mathrm{I}_{2}$ has been plotted as a function of polymer concentration in Figure 1 and the CMC of Poloxamer 407/TPGS binary mixture was found to be $0.0013 \%$ w/v. The low CMC of the micelles suggests high stability and ability to maintain integrity even upon dilution in the blood circulation.

\section{Particle size distribution}

To achieve longevity during systemic circulation, the micelles must be small enough to evade detection and destruction by the reticulo-endothelial system (RES). The average micelle size and the unimodal size distribution of both empty and GA-loaded Poloxamer 407/TPGS mixed micelles are illustrated in Figure $2 \mathrm{~A}$ and $\mathrm{B}$, indicating the formation of nanosized mixed micelles. The mean diameter of blank micelles as well as GA-loaded Poloxamer 407/TPGS mixed micelles was close to $16.6 \pm 0.1 \mathrm{~nm}$ and $17.4 \pm 0.5 \mathrm{~nm}$ respectively, with an acceptable polydispersity index between $0.1-0.2$. Encapsulating the GA in the micelles

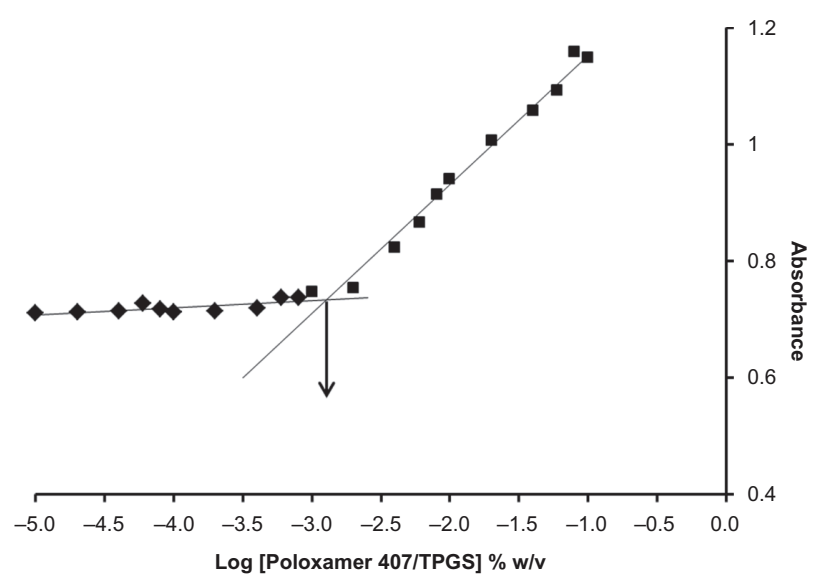

Figure I Plot of ultraviolet absorbance of $\mathrm{I}_{2}$ versus concentration of Poloxamer 407/TPGS mixed micelles (molar ratio 3:7) in water. Critical micelle concentration value was calculated by corresponding polymer concentration at which a sharp increase in absorbance is observed.

Abbreviation: TPGS, D- $\alpha$-Tocopheryl polyethylene glycol 1000 succinate. 

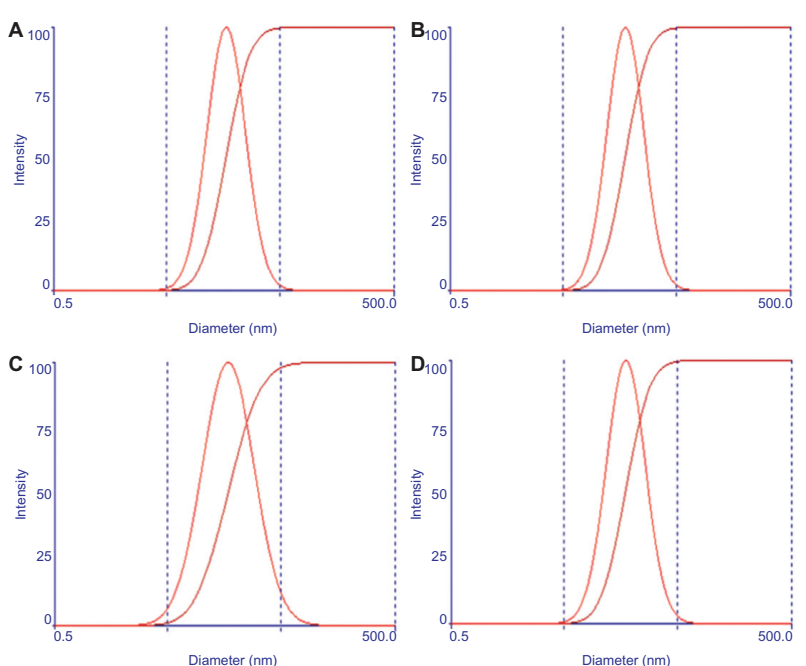

Figure 2 Particle size distribution of blank micelles ( $\mathbf{A}$ and $\mathbf{C})$ and gambogic acid-loaded Poloxamer 407/TPGS mixed micelles (B and D), before freeze drying ( $\mathbf{A}$ and $\mathbf{B})$ and after freeze drying (C and D). The x-axis is in logarithmic scale from 0.5 to 500 (the middle two points on the $x$-axis shown by the dashed line are 5 and $50 \mathrm{~nm}$, respectively. Abbreviation: TPGS, D- $\alpha$-Tocopheryl polyethylene glycol 1000 succinate.

did not increase the micelle average size and size distribution. It was found that there was no increase in the particle size of empty and GA-loaded Poloxamer 407/TPGS mixed micelles after freeze drying (Figure $2 \mathrm{C}$ and D). After 6 months in storage at $4^{\circ} \mathrm{C}$, the drug content of freeze dried GA-loaded Poloxamer 407/TPGS mixed micelles did not change, indicating that lyophilization is a good choice for storage of mixed micelles.

\section{Zeta potential}

Both empty and GA-loaded Poloxamer 407/TPGS mixed micelles were negatively charged with zeta potential of about $-8.52 \mathrm{mV}$ and $-13.57 \mathrm{mV}$, respectively. In the structure of Poloxamer 407 both the polypropylene oxide and polyethylene oxide segments were nonionic, so the change of the surface charge of the micelles should result from addition of GA and/orTPGS. The free carboxylic group of GA can dissociate and result in the anionic property for GA. Therefore, the absolute value of zeta potential was increased by addition of drug. The increased negative surface charge of GA might be attributed to TPGS, which enhanced the solubility of GA and more anionic GA was present in solution.

\section{Drug loading and encapsulation efficiency}

DL $\%$ of the GA-loaded Poloxamer 407/TPGS mixed micelles was about $9.38 \% \pm 0.29 \%$ and the $\mathrm{EE} \%$ was $93.1 \% \pm 0.5 \%$. The encapsulation of GA in the mixed micelle system is high at this drug loading.

\section{DSC and XRPD analysis}

The physical status of GA inside the micelles was examined by DSC and XRPD. DSC thermogram (Figure 3A) revealed endothermic peaks of GA and Poloxamer 407/TPGS. Thermogram for physical mixture shows all the endothermic peaks of GA and Poloxamer/TPGS while the thermogram for blank Poloxamer 407/TPGS mixed micelles only shows endothermic peaks of polymers. The thermogram of GAloaded Poloxamer 407/TPGS micelles shows all endothermic peaks of polymers as shown in the blank micelles thermogram, but the characteristic peaks (at $140^{\circ} \mathrm{C}$ ) of $\mathrm{GA}$ was absent, which confirmed the encapsulation of GA inside the Poloxamer 407/TPGS micelles.

XRPD diffraction spectra (Figure 3B) shows characteristic diffraction peaks of GA, which were visible in the pattern obtained for GA as well as for the physical mixture of GA, Poloxamer 407, and TPGS, but disappeared in the pattern of GA-loaded Poloxamer 407/TPGS, especially around $4^{\circ} \mathrm{C}-10^{\circ} \mathrm{C}$. This indicates that GA was encapsulated in the polymeric micelles in molecular or amorphous state and there was no free drug on the surface of micelles.
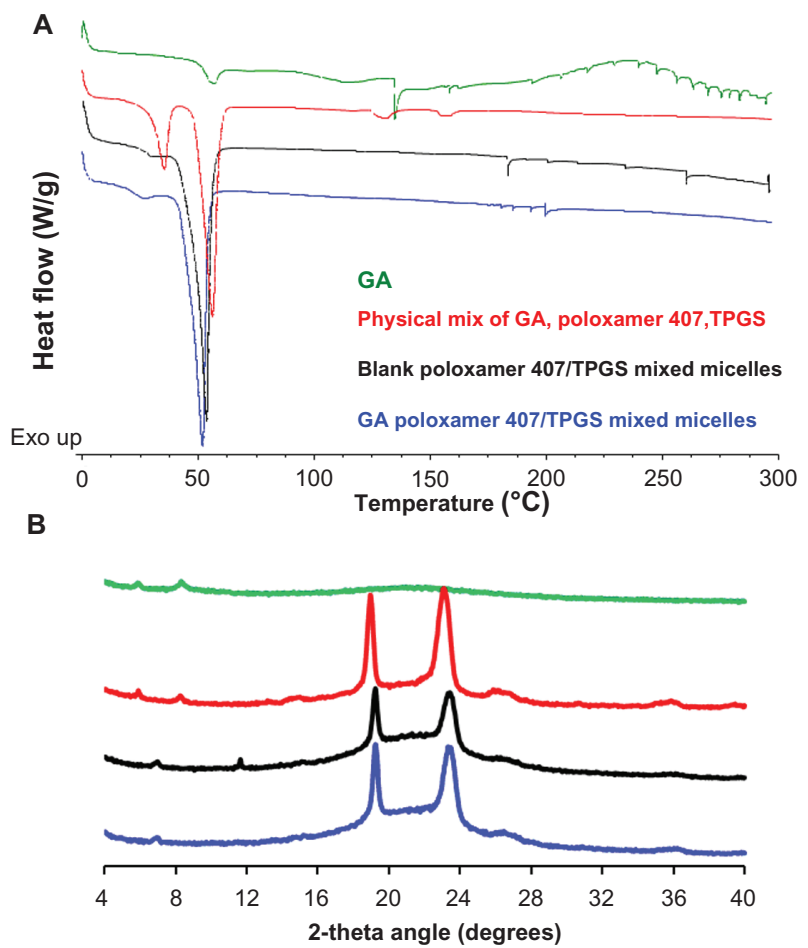

Figure 3 (A) Differential scanning calorimetric thermogram and (B) X-ray powder diffraction spectra of gambogic acid (GA) (green lines), physical mixture of GA, Poloxamer 407 and TPGS (red lines), blank poloxamer/TPGS mixed micelles (Black lines) and GA-loaded poloxamer/TPGS mixed micelles (blue lines).

Abbreviations: GA, gambogic acid; TPGS, D- $\alpha$-Tocopheryl polyethylene glycol 1000 succinate. 


\section{In vitro drug release}

The in vitro release of GA from mixed micellar formulation under sink conditions was investigated by dialysis method with $0.5 \%$ Tween 80 solution as release medium. As shown in Figure 4, about 30\% of GA was released from mixed micelles within the first 4 hours, while almost $100 \%$ of GA was released from the GA solution during the same time period. After 4 days approximately $90 \%$ of the GA was released from GA-loaded Poloxamer 407/TPGS mixed micelles. The micellar carrier can not only solubilized the poorly soluble GA, but also sustained the GA release for more than 4 days. Drug diffusion, polymer erosion or swelling might be possible mechanisms for release of GA from micelles. ${ }^{32}$

\section{Cellular uptake study}

To evaluate the cellular internalization of GA-loaded Poloxamer 407/TPGS mixed micelles, the cellular uptake by MCF-7 and NCI/ADR-RES cells were investigated. Figure 5A1-3 and B1-3 shows the CLSM images of free GA and GA-loaded Poloxamer 407/TPGS mixed micelles respectively, in MCF-7 cells. GA itself was fluorescent under the CLSM and the images show that, similar to free GA, GA-loaded Poloxamer 407/TPGS mixed micelles also distributed throughout the cytoplasm. This indicates that the mixed micelles have been taken up by the cells and are located inside the cells. There is equal or higher uptake of GA-loaded Poloxamer 407/TPGS mixed micelles in MCF-7.

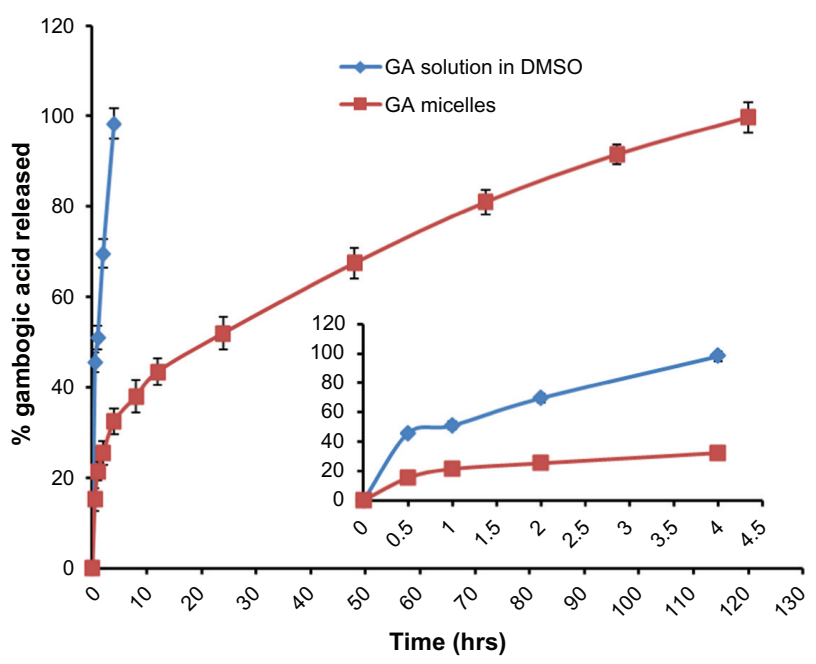

Figure 4 In vitro release profile of gambogic acid (GA)-loaded Poloxamer 407/TPGS mixed micelles and GA solution in DMSO. Drug release study was performed at $37^{\circ} \mathrm{C}$ under shaking ( $100 \mathrm{rpm})$ which was monitored using a dialysis bag containing phosphatebuffered saline with addition of $0.5 \%$ Tween- 80 as a sink solution at $\mathrm{pH} 7.4$.

Notes: Mean $\pm S D, n=3$.

Abbreviations: DMSO, dimethyl sulfoxide; GA, gambogic acid; TPGS, $D-\alpha-$ Tocopheryl polyethylene glycol 1000 succinate; SD, standard deviation.

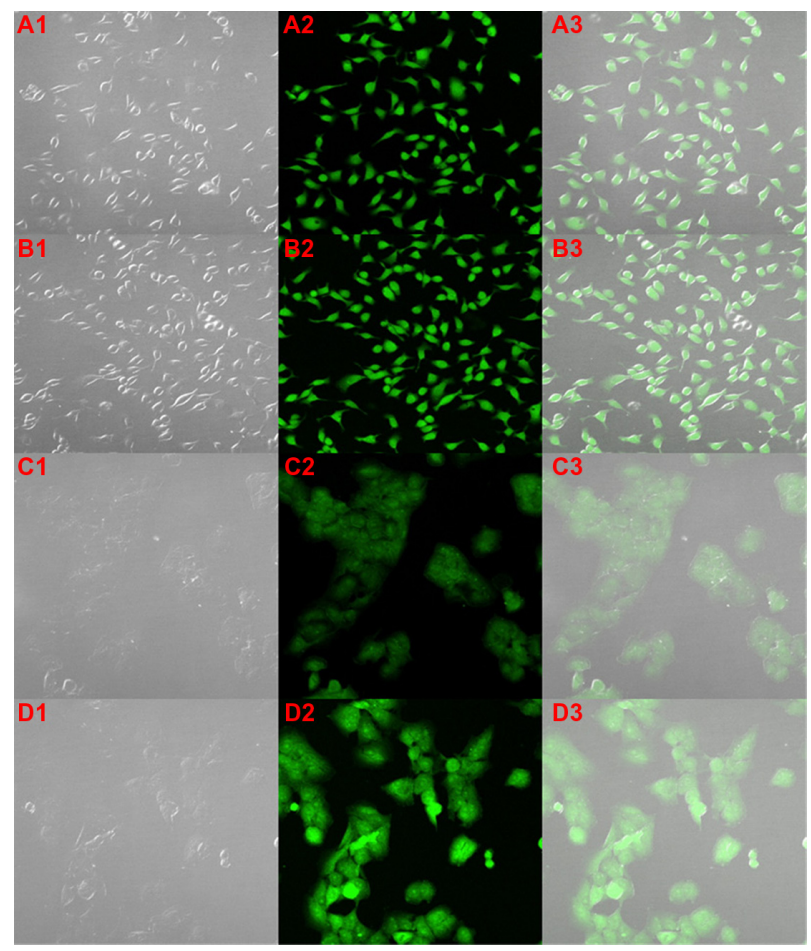

Figure 5 Cellular internalization of gambogic acid (GA) (A and $\mathbf{C}$ ) and GA-loaded poloxamer/TPGS mixed micelles ( $\mathbf{B}$ and $\mathbf{D}$ ) by confocal laser scanning microscopy in breast cancer MCF-7 cells ( $\mathbf{A}$ and $\mathbf{B}$ ) and multidrug resistant NCl/ADR-RES cells (C and D) after 4 hours of incubation. Images show an increased uptake of GA-loaded micelles inside the multidrug resistant NCI/ADR-RES cells.

Abbreviations: TPGS, D- $\alpha$-Tocopheryl polyethylene glycol 1000 succinate.

The uptake of free GA may be due to the binding affinity of GA for transferrin receptors ${ }^{33}$ while the micelles are internalized by endocytosis. Figure 5C1-3 and D1-3 show the CLSM images of free GA and GA-loaded Poloxamer 407/TPGS mixed micelles respectively, in NCI/ADR-RES cells. It was clear that the intensity of the green fluorescence inside the cells treated with GA-loaded Poloxamer 407/TPGS mixed micelles (Figure 5D) was much greater than that of the free GA (Figure 5C). This indicates an enhanced uptake of GA-loaded Poloxamer 407/TPGS mixed micelles in NCI/ADR-RES cells as compared to free GA. NCI/ADR-RES cells expressing the P-gp may have higher efflux of GA from the cells, but mixed micelles taken up by cells via endocytosis are not subject to efflux by P-gp. Also, Poloxamer 407/TPGS mixed micelles may inhibit the P-gp efflux pump..$^{34,35}$

\section{In vitro cytotoxicity study}

Figure 6A shows the in vitro cytotoxic effect of free GA and GA-loaded Poloxamer 407/TPGS mixed micelles in MCF-7, and Figure 6B shows it in NCI/ADR-RES cells. The results indicate that GA formulated in the polymeric micelles has 
shown advantages in achieving higher cytotoxicity than the free GA (Figure 7). The $\mathrm{IC}_{50}$ of GA-loaded Poloxamer 407/ TPGS mixed micelles $(0.44 \pm 0.02 \mu \mathrm{M})$ in MCF-7 cells which is about 1.6 fold less than the free GA $(0.71 \pm 0.06 \mu \mathrm{M})$ $(P<0.05)$ and the $\mathrm{IC}_{50}$ of GA-loaded Poloxamer 407/TPGS mixed micelles $(0.86 \pm 0.01 \mu \mathrm{M})$ in NCI/ADR-RES cells was found to be 2.9 fold less than the free GA $(2.5 \pm 0.08 \mu \mathrm{M})$ $(P<0.01)$. The lower cytotoxicity of unencapsulated GA may be due to its efflux by P-gp pumps. On the other hand, GA-loaded Poloxamer 407/TPGS mixed micelles are taken up by cells via endocytosis, and are not subject to efflux by P-gp. This allows sustaining the drug concentration inside the cells which results in a higher cytotoxic effect, which is also reported in the literature. ${ }^{30,36,37}$ Another explanation for improved cytotoxicity of GA-loaded mixed micelles was the effect of Poloxamer 407 and TPGS, which are well known P-gp inhibitors and also reduce drug efflux. ${ }^{34,35}$ Empty Poloxamer 407/TPGS mixed micelles had no significant effects on the viability of MCF-7 and NCI/ADR-RES cells at concentrations up to $17.4 \mu \mathrm{g} / \mathrm{mL}$ (Figure 6C), which is much higher than the concentration needed for $\mathrm{IC}_{50}$ of GAloaded mixed micelles. The anticancer activity of TPGS is reported to be related to its unique apoptosis-inducing properties via the generation of reactive oxygen species. This might be the reason for toxicity of empty mixed micelles to cancer cells at a higher concentration. ${ }^{38,39}$ The P-gp inhibition by the Poloxamer 407/TPGS mixed micelles makes these micelles a better nanocarrier than other micelles such as PEG-PCL ${ }^{40}$ and chitosan micelles ${ }^{41}$ to treat multi-drug resistant cancer.

\section{Conclusion}

The purpose of this study was to design mixed micelles composed of Poloxamer 407 and TPGS loaded with the poorly soluble natural anticancer agent, GA. The micelles have a low CMC, small particle size and high encapsulation efficiency. GA release was sustained as a result of encapsulation into the inner core of the micelles. GA encapsulation by Poloxamer 407/TPGS mixed micelles also demonstrated an equal or increased cellular uptake and increased in vitro cytotoxicity compared to free GA in breast cancer cells MCF-7 as well as in multidrug resistant NCI/ADR-RES cells. Results suggested that GA encapsulated Poloxamer 407/TPGS mixed micelles will be a good nanoformulation to treat breast and multidrug-resistant cancer. Future studies will be focus on in vivo evaluation of GA-loaded Poloxamer 407/TPGS mixed micelles.

\section{Disclosure}

The authors report no conflicts of interest in this work.
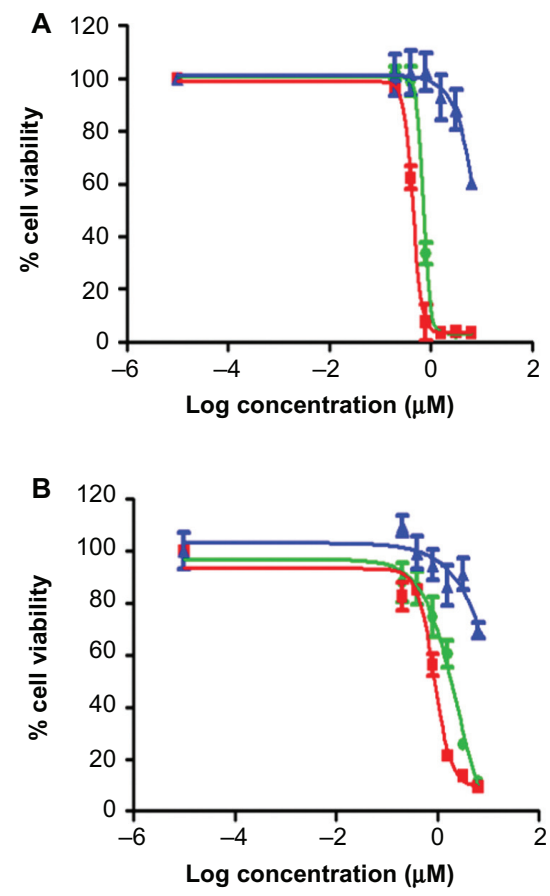

C

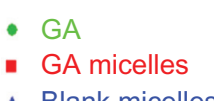

- Blank micelles

Figure 6 In vitro cytotoxicity of free gambogic acid (GA), GA-loaded Poloxamer 407/TPGS mixed micelles, blank poloxamer/TPGS mixed micelles in (A) breast cancer MCF-7 cells and (B) multidrug resistant NCI/ADR-RES cells after 72 hours of incubation. (C) Cytotoxicity of different concentrations of blank poloxamer/TPGS mixed micelles in breast cancer MCF-7 cells and multidrug-resistant NCl/ADR-RES cells after 72 hours of incubation.

Notes: Mean \pm SD, $\mathrm{n}=3$.

Abbreviations: TPGS, D- $\alpha$-Tocopheryl polyethylene glycol 1000 succinate; SD, standard deviation. 


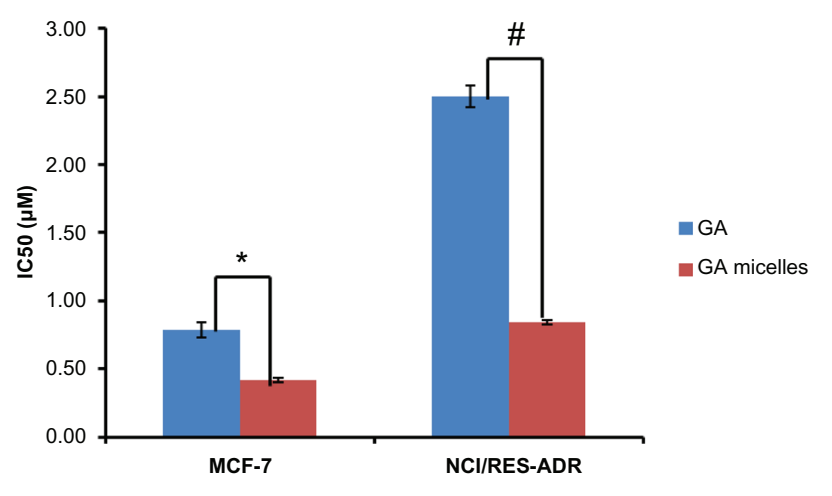

Figure $7 I_{50}$ values of free gambogic acid (GA) and GA-loaded Poloxamer 407/ TPGS mixed micelles in breast cancer MCF-7 cells and multidrug resistant $\mathrm{NCl}$ / ADR-RES cells after 72 hours of incubation.

Notes: Data shown as mean $\pm \mathrm{SD}, \mathrm{n}=3$ separate experiments. $* P<0.05,{ }^{\#} P<0.01$ Abbreviations: TPGS, D- $\alpha$-Tocopheryl polyethylene glycol 1000 succinate; SD, standard deviation.

\section{References}

1. Ross DD. Novel mechanisms of drug resistance in leukemia. Leukemia. 2000;14(3):467-473.

2. Liu F, Xie ZH, Cai GP, Jiang YY. The effect of survivin on multidrug resistance mediated by $\mathrm{P}$-glycoprotein in $\mathrm{MCF}-7$ and its adriamycin resistant cells. Biol Pharm Bull. 2007;30(12):2279-2283.

3. Alakhov VYu, Moskaleva EYu, Batrakova EV, Kabanov AV. Hypersensitization of multidrug resistant human ovarian carcinoma cells by pluronic P85 block copolymer. Bioconjug Chem. 1996;7(2): 209-216.

4. Batrakova E, Lee S, Li S, Venne A, Alakhov V, Kabanov A. Fundamental relationships between the composition of pluronic block copolymers and their hypersensitization effect in MDR cancer cells. Pharm Res. 1999; 16(9):1373-1379.

5. Jang SW, Okada M, Sayeed I, et al. Gambogic amide, a selective agonist for TrkA receptor that possesses robust neurotrophic activity, prevents neuronal cell death. Proc Natl Acad Sci U S A. 2007;104(41): 16329-16334.

6. Panthong A, Norkaew P, Kanjanapothi D, Taesotikul T, Anantachoke N, Reutrakul V. Anti-inflammatory, analgesic and antipyretic activities of the extract of gamboge from Garcinia hanburyi Hook $\mathrm{f}$. J Ethnopharmacol. 2007;111(2):335-340.

7. Gu H, Rao S, Zhao J, et al. Gambogic acid reduced bcl-2 expression via p53 in human breast MCF-7 cancer cells. J Cancer Res Clin Oncol. 2009;135(12):1777-1782.

8. Zhao L, Guo QL, You QD, Wu ZQ, Gu HY. Gambogic acid induces apoptosis and regulates expressions of $\mathrm{Bax}$ and $\mathrm{Bcl}-2$ protein in human gastric carcinoma MGC-803 cells. Biol Pharm Bull. 2004;27(7):998-1003.

9. Liu W, Guo QL, You QD, Zhao L, Gu HY, Yuan ST. Anticancer effect and apoptosis induction of gambogic acid in human gastric cancer line BGC-823. World J Gastroenterol. 2005;11(24):3655-3659.

10. Guo QL, You QD, Wu ZQ, Yuan ST, Zhao L. General gambogic acids inhibited growth of human hepatoma SMMC-7721 cells in vitro and in nude mice. Acta Pharmacol Sin. 2004;25(6):769-774.

11. Yi T, Yi Z, Cho SG, et al. Gambogic acid inhibits angiogenesis and prostate tumor growth by suppressing vascular endothelial growth factor receptor 2 signaling. Cancer Res. 2008;68(6):1843-1850.

12. Chen J, Gu HY, Lu N, et al. Microtubule depolymerization and phosphorylation of c-Jun N-terminal kinase-1 and p38 were involved in gambogic acid induced cell cycle arrest and apoptosis in human breast carcinoma MCF-7 cells. Life Sci. 2008;83(3-4):103-109.

13. Yu J, Guo QL, You QD, et al. Repression of telomerase reverse transcriptase mRNA and hTERT promoter by gambogic acid in human gastric carcinoma cells. Cancer Chemother Pharmacol. 2006;58(4): 434-443.
14. Qi Q, Gu H, Yang Y, et al. Involvement of matrix metalloproteinase 2 and 9 in gambogic acid induced suppression of MDA-MB-435 human breast carcinoma cell lung metastasis. J Mol Med (Berl). 2008;86(12): 1367-1377.

15. Wang T, Wei J, Qian X, Ding Y, Yu L, Liu B. Gambogic acid, a potent inhibitor of survivin, reverses docetaxel resistance in gastric cancer cells. Cancer Lett. 2008;262(2):214-222.

16. Yang Y, Yang L, You QD, et al. Differential apoptotic induction of gambogic acid, a novel anticancer natural product, on hepatoma cells and normal hepatocytes. Cancer Lett. 2007;256(2):259-266.

17. Qu G, Zhu X, Zhang C, Ping Q. Modified chitosan derivative micelle system for natural anti-tumor product gambogic acid delivery. Drug Deliv. 2009;16(7):363-370.

18. Gelderblom H, Verweij J, Nooter K, Sparreboom A. Cremophor EL: the drawbacks and advantages of vehicle selection for drug formulation. Eur J Cancer. 2001;37(13):1590-1598.

19. Cho H, Kwon GS. Polymeric Micelles for Neoadjuvant Cancer Therapy and Tumor-Primed Optical Imaging. ACS Nano. 2011;5(11): 8721-8729.

20. Shin HC, Alani AW, Rao DA, Rockich NC, Kwon GS. Multi-drug loaded polymeric micelles for simultaneous delivery of poorly soluble anticancer drugs. J Control Release. 2009;140(3):294-300.

21. Oerlemans C, Bult W, Bos M, Storm G, Nijsen JF, Hennink WE. Polymeric micelles in anticancer therapy: targeting, imaging and triggered release. Pharm Res. 2010;27(12):2569-2589.

22. Yokoyama M. Polymeric micelles as a new drug carrier system and their required considerations for clinical trials. Expert Opin Drug Deliv. 2010;7(2):145-158.

23. Torchilin VP. Structure and design of polymeric surfactant-based drug delivery systems. J Control Release. 2001;73(2-3):137-172.

24. Maeda H, Bharate GY, Daruwalla J. Polymeric drugs for efficient tumortargeted drug delivery based on EPR-effect. Eur J Pharm Biopharm. 2009;71(3):409-419.

25. Oh KT, Bronich TK, Kabanov AV. Micellar formulations for drug delivery based on mixtures of hydrophobic and hydrophilic Pluronic block copolymers. J Control Release. 2004;94(2-3):411-422.

26. Zhang W, Shi Y, Chen Y, Ye J, Sha X, Fang X. Multifunctional Pluronic P123/F127 mixed polymeric micelles loaded with paclitaxel for the treatment of multidrug resistant tumors. Biomaterials. 2011;32(11): 2894-2906.

27. Mi Y, Liu Y, Feng SS. Formulation of Docetaxel by folic acidconjugated d-alpha-tocopheryl polyethylene glycol succinate 2000 (Vitamin ETPGS(2k)) micelles for targeted and synergistic chemotherapy. Biomaterials. 2011;32(16):4058-4066.

28. Mu L, Elbayoumi TA, Torchilin VP. Mixed micelles made of poly(ethylene glycol)-phosphatidylethanolamine conjugate and d-alphatocopheryl polyethylene glycol 1000 succinate as pharmaceutical nanocarriers for camptothecin. Int J Pharm. 2005;306(1-2): 142-149.

29. Gao ZG, Fain HD, Rapoport N. Controlled and targeted tumor chemotherapy by micellar-encapsulated drug and ultrasound. J Control Release. 2005;102(1):203-222.

30. Wei Z, Hao J, Yuan S, et al. Paclitaxel-loaded Pluronic P123/ F127 mixed polymeric micelles: formulation, optimization and in vitro characterization. Int J Pharm. 2009;376(1-2):176-185.

31. Zhao L, Shi Y, Zou S, Sun M, Lil L, Zhail G. Formulation and in vitro evaluation of quercetin loaded polymeric micelles composed of pluronic P123 and D-a-tocopheryl polyethylene glycol succinate. J Biomed Nanotechnol. 2011;7(3):358-365.

32. Kim KS, Park SJ. Effect of porous silica on sustained release behaviors of pH sensitive pluronic F127/poly(acrylic acid) hydrogels containing tulobuterol. Colloids Surf B Biointerfaces. 2010;80(2):240-246.

33. Kasibhatla S, Jessen KA, Maliartchouk S, et al. A role for transferrin receptor in triggering apoptosis when targeted with gambogic acid. Proc Natl Acad Sci U S A. 2005;102(34):12095-12100.

34. Guan Y, Huang J, Zuo L, et al. Effect of pluronic P123 and F127 block copolymer on P-glycoprotein transport and CYP3 A metabolism. Arch Pharm Res. 2011;34(10):1719-1728. 
35. Collnot EM, Baldes C, Wempe MF, et al. Mechanism of inhibition of P-glycoprotein mediated efflux by vitamin ETPGS: influence on ATPase activity and membrane fluidity. Mol Pharm. 2007;4(3):465-474.

36. Panyam J, Zhou WZ, Prabha S, Sahoo SK, Labhasetwar V. Rapid endo-lysosomal escape of poly(DL-lactide-co-glycolide) nanoparticles: implications for drug and gene delivery. FASEB J. 2002;16(10): 1217-1226.

37. Gabizon AA. Selective tumor localization and improved therapeutic index of anthracyclines encapsulated in long-circulating liposomes. Cancer Res. 1992;52(4):891-896.

38. Youk HJ, Lee E, Choi MK, et al. Enhanced anticancer efficacy of alpha-tocopheryl succinate by conjugation with polyethylene glycol. J Control Release. 2005;107(1):43-52.
39. Liu Y, Huang L, Liu F. Paclitaxel nanocrystals for overcoming multidrug resistance in cancer. Mol Pharm. 2010;7(3):863-869.

40. Diao YY, Li HY, Fu YH, et al. Doxorubicin-loaded PEG-PCL copolymer micelles enhance cytotoxicity and intracellular accumulation of doxorubicin in adriamycin-resistant tumor cells. Int J Nanomedicine. 2011;6:1955-1962.

41. Xu YY, Du YZ, Yuan H, Liu LN, Niu YP, Hu FQ. Improved cytotoxicity and multidrug resistance reversal of chitosan based polymeric micelles encapsulating oxaliplatin. J Drug Target. 2011;19(5):344-353.
International Journal of Nanomedicine

\section{Publish your work in this journal}

The International Journal of Nanomedicine is an international, peerreviewed journal focusing on the application of nanotechnology in diagnostics, therapeutics, and drug delivery systems throughout the biomedical field. This journal is indexed on PubMed Central,

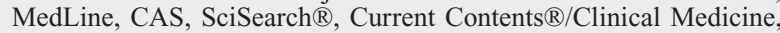

\section{Dovepress}

Journal Citation Reports/Science Edition, EMBase, Scopus and the Elsevier Bibliographic databases. The manuscript management system is completely online and includes a very quick and fair peer-review system, which is all easy to use. Visit http://www.dovepress.com/ testimonials.php to read real quotes from published authors.

Submit your manuscript here: http://www.dovepress.com/international-journal-of-nanomedicine-journal 